\title{
Black Sea Catchment Observation System as a Portal for GEOSS Community
}

\author{
Dorian Gorgan ${ }^{1}$, Gregory Giuliani ${ }^{2,7}$, Nicolas Ray ${ }^{2,7}$, Anthony Lehmann ${ }^{2}$, Pierluigi Cau ${ }^{3}$, \\ Karim Abbaspour ${ }^{4}$, Karel Charvat ${ }^{5}$, Andreja Jonoski ${ }^{6}$ \\ ${ }^{1}$ Computer Science Department, Technical University of Cluj-Napoca, Cluj-Napoca, Romania, dorian.gorgan@cs.utcluj.ro \\ ${ }^{2}$ EnviroSPACE Laboratory, University of Geneva, Geneva, Switzerland \\ gregory.giuliani@unepgrid.ch,nicolas.ray@unige.ch, anthony.lehmann@unige.ch \\ ${ }^{3}$ CRS4 - Center for Advanced Studies Research, Sardinia, Italy, pierluigi.cau@gmail.com \\ ${ }^{4}$ EAWAG Institute of Aquatic Science and Technology, Zurich, Switzerland, karim.abbaspour@eawag.ch \\ ${ }^{5}$ CCSS - Czech Centre for Science and Society, Prague, Czech Republic, charvat@ccss.cz \\ ${ }^{6}$ UNESCO-IHE Institute for Water Education, Delft, The Netherlands, a.jonoski@unesco-ihe.org \\ ${ }^{7}$ United Nations Environment Programme Global Resource Information Database, 1211 Châtelaine, Switzerland
}

\begin{abstract}
The resources of the enviroGRIDS system are accessible to the large community of users through the BSCOS Portal that provides Web applications for data management, hydrological model calibration and execution, satellite image processing, report generation and visualization, citizens oriented applications, and virtual training center. The portal publishes through Internet both the geospatial functionality provided by Web technologies, and the high power computation resources supported by the Grid technologies. The paper highlights the issues on the implementation of the portal by heterogeneous technologies, in order to support control flow, processing, and visualization of spatial data for GEOSS community, Earth Science specialists, and generally for Web users.
\end{abstract}

Keywords-Grids computing; geospatial; SWAT hydrological model; satellite image processing; spatial data processing; distributed computing.

\section{INTRODUCTION}

To study and to search solutions for improvement of the sustainable development of environment and adequate resource management in the Black Sea catchment region, are ones of the main objectives of the enviroGRIDS (Black Sea Catchment Observation and Assessment System supporting Sustainable Development) project [1]. Moreover the evolution of the complex environmental systems is analyzed in context of land cover, demographic, industrial, and climate changes. One of the main goals of this project is to simulate environmental scenarios concerning the quantity and quality of waters over the coming decades. The enviroGRIDS project aims to develop, calibrate, and provide for execution hydrological models of the Black Sea catchment region. There are four main tasks carried out by the project consortium:

1) Collect environmental sets of data regarding the Black Sea catchment region;

2) Develop a dedicated Spatial Data Infrastructure (SDI) in order to support data sharing and distributed processing;

3) Calibrate and execute high-resolution and large area hydrological models on distributed infrastructures such as Grid;

4) Provide tools and applications to specialists, decision makers, and citizens in order to access data processing and visualization, and develop and run environmental scenarios.

The enviroGRIDS project aims to put together different software technologies and heterogeneous computing resources. One of the main issues of the project is to develop solutions based on interoperability between different technologies, platforms, and applications. For instance, such a case is the interoperability between the Geospatial and Grid infrastructures, in order to get benefits by using in a collaborative manner the both technologies. Each of them comes with important features. While the Geospatial platforms provides very specialized functionalities for Earth Science oriented applications, the Grid infrastructures support scalable, distributed, and parallel high performance computation.

The tools, applications, platforms, and resources of the enviroGRIDS system are available to wide communities of users through its Web portal, called BSC-OS (Black Sea Catchment - Observation System). The system provides graphical user interfaces to Web applications for data management, hydrological model calibration and execution, satellite image processing, report generation and visualization, environmental scenarios, and virtual training center. In order to simplify the access to all these tools and applications the portal has implemented the single sign-on authentication mechanism, through which the user has to authenticate just one times, and then gets authorization to all resources during the same working session.

This paper highlights the availability of the BSC-OS portal, by its resources, tools, applications, and platforms to GEOSS community, Earth Science specialists, and generally to internet users. Meanwhile the presentation focuses on the main challenges and issues regarding the development and using of the BSC-OS portal.

The presentation is structured as follows. Section II presents the works and achievements related with the enviroGRIDS project. Section III describes GEOSS components and services. Section IV sketches the BSC-OS portal architecture and the set of tool and application categories. Each of the next six sections V-X describes a tool and application category such as data management, SWAT model calibration and scenario execution by gSWAT application, satellite image 
processing by GreenLand application, geospatial data visualization, two flood scenarios addressing citizens and decision makers, and training material development and execution. The last section XI concludes on the portal development and future work.

\section{RELATED WORKS}

Black Sea catchment region is a huge geographical area and a very complex environment. The watershed related hydrological model involves highly interconnected and continuously evolving interactions at many spatial and temporal scales, and requires to gather and integrate different sets of environmental data such as physical, chemical, and biological [2]. The enviroGRIDS project managed to develop and calibrate the SWAT model [3] as a high-resolution water balance model to the entire Black Sea catchment region, by sub-catchment spatial and daily temporal resolution. The model has been calibrated and validated by using river discharge data, river water quality data, and crop yield data by the approach described in [4]. There are many other projects that are used the SWAT model for limited hydrological areas. The enviroGRIDS project is the first attempt that has accomplished to build and calibrate a such huge hydrological model for the Black Sea catchment region and the Danube River.

There are two other hydrological models such as HECHMS and SOBEK that are also used to develop use cases for limited regions. HEC-HMS (Hydrologic Engineering Center Hydrological Modeling System) [5] is a generic modeling system for simulating precipitation-runoff processes in dendritic catchments. The catchment under study is usually divided in a number of sub-catchments with spatially varying parameters and meteorological inputs. Runoff generation is computed for each sub-catchment and subsequently the generated runoff is routed downstream to the catchment outlet. SOBEK modeling system [6] is used for setting-up the flooding and sediment transport model. SOBEK is a software tool used for flow modeling in many areas such as irrigation systems, drainage systems, and natural streams. The SOBEK 1D/2D model combines one-dimensional (1D) hydraulic modeling of the river channel to a two-dimensional (2D) representation of the floodplains. The enviroGRIDS project has used the HEC-HMS and SOBEK models to study two flood forecasting scenarios in Romania.

Other European projects deal with environmental related subjects [7]. Each project is focused on a specific domain and user community of Earth Science. DRIHM (Distributed Research Infrastructure for Hydro-Meteorology) project [8] attempts to improve the use of Grid and HPC (High Performance Computing) just for HMR Hydro-Meteorological Research) modeling and observational databases. The project supports the study of severe hydrometeorological events, the execution and analysis of high-end simulations, and the dissemination of predictive models as decision analysis tools. ENES and ENSEMBLES projects worked on understanding and prediction of future climate change based on the high resolution, global and regional Earth System models developed in Europe. METAFOR and GENESI-DEC projects aim to provide effective access of users to a variety of data repositories, facilities, tools and services. Other European projects such as SAWGEO, CYCLOPS, GDI-Grid, GEO-Grid, DEEGREE, DORII, and GENESI-DR address the management of spatial data and environmental tools and applications. EGEE, SEEGRID-SCI, and $\mathrm{C} 3 \mathrm{Grid}$ projects have developed Grid based solutions for sharing complex spatial and environmental data sets. Other EU projects such as OBSERVE, EGIDA, Balkan GEONET, BalkanGEONet, and GEONetCab have significant contribution to the development of the environmental network and observation capacity in the South East Europe.

Now, other projects are working on software technologies for developing tools, services, and infrastructures, that can be used by the Earth Science and Environmental community. ENVRI project [9] is a collaboration in the ESFRI (European Strategy Forum on Research Infrastructures) Environmental Cluster, to develop common e-science software components and services. The results will speed up the construction of environmental infrastructures and will allow scientists to use the data and software from each facility to enable multidisciplinary science. LifeWatch [10] is a European research infrastructure aiming the biodiversity and ecosystem research. Users benefit from integrated access to a variety of data, analytical and modelling tools provided by a variety of collaborating initiatives. LifeWatch offers data and tools in selected workflows for specific scientific communities, and provides as well possibility to construct personalized virtual laboratories, including new data and analytical tools.

The enviroGRIDS project [11] has concerned with Earth Science and Grid based solutions approaching the particularities of the Black Sea catchment region in order to:

- Collect and build a dedicated SDI that is able to support data sharing and distributed processing;

- Process over the Grid infrastructure the huge spatial data such as hydrological models, satellite images, and maps;

- Support scalability in terms of high number of users, applications, and models, high model resolution, large areas, and big dimension of data models;

- Develop interactive applications which hide the complexity of the computation infrastructure and the huge data management. Provide all these applications by the BSC-OS portal to the Earth Science community;

- Calibrate the huge SWAT models of the Black Sea Catchment region and Danube River.

- Process great number of satellite images over the Grid infrastructure;

- Develop training materials by including Earth Science dynamical content and Grid based processing.

- Support interoperability between the Geospatial and Grid platforms, and compatibility of software platforms like URM (Uniform Resource Management), gSWAT, ESIP, GreenLand, gProcess, CWE (Collaborative Work Environment), and eGLE.

\section{GEOSS COMPONENTS AND SERVICES}

The main tools and applications from the enviroGRIDS portal are registered into the GEO Portal [12] and available as GEOSS services to the large community of Web users. 


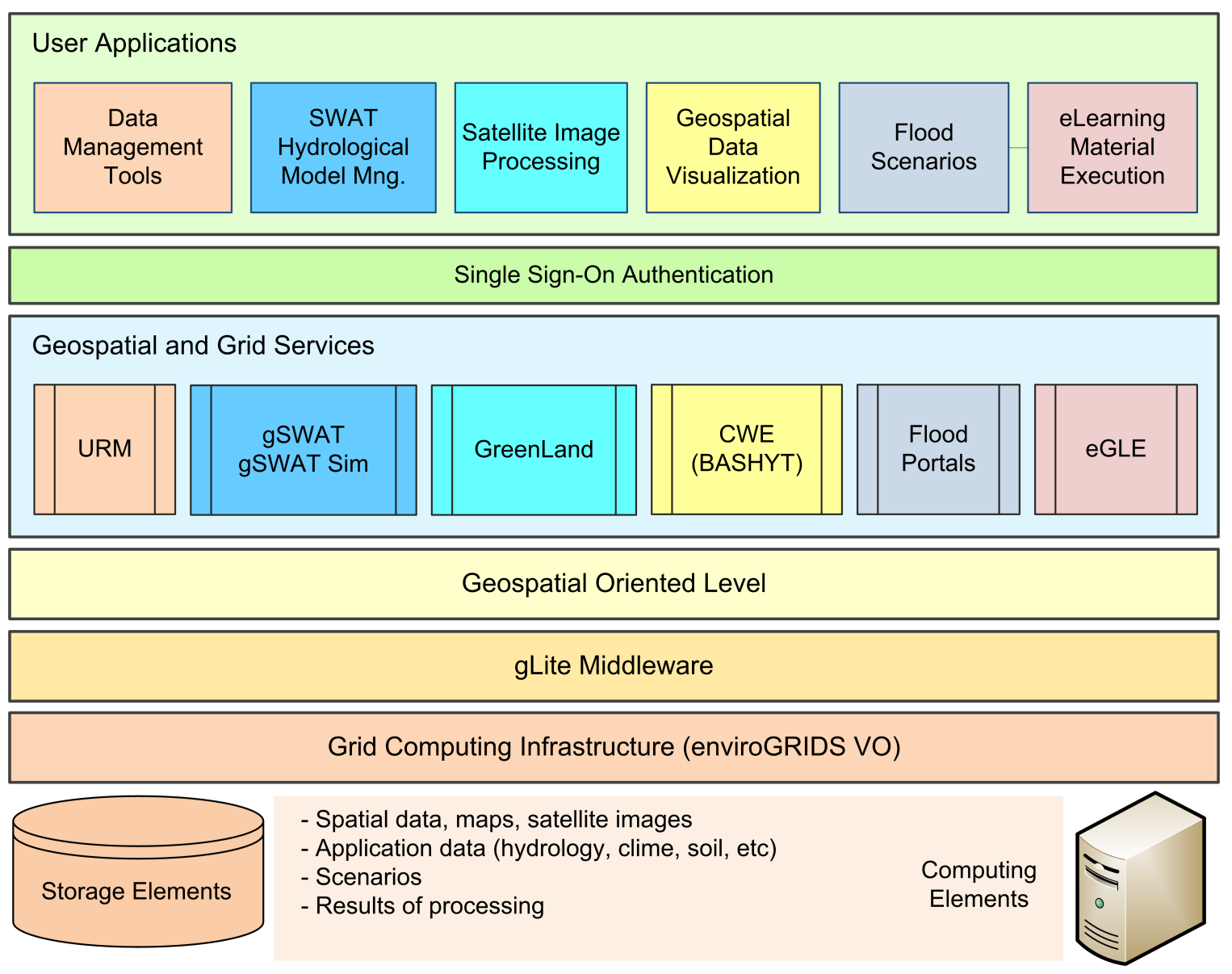

Figure 1. BSC-OS portal architecture

GEOSS (Global Earth Observation System of Systems) [13] is an achievement of the Group on Earth Observations (GEO). The intention of GEOSS is to build Web based global and flexible network compose of content providers. The main idea is to guarantee access of decision makers and a large community of users to range of information. GEOSS is intended to be a system of systems, which will proactively link together existing and planned observing systems around the world and support the development of new systems where gaps currently exist.

GEOSS is simultaneously addressing nine areas of critical importance to people and society. It aims to empower the international community to protect itself against natural and humaninduced disasters, understand the environmental sources of health hazards, manage energy resources, respond to climate change and its impacts, safeguard water resources, improve weather forecasts, manage ecosystems, promote sustainable agriculture and conserve biodiversity. The GEOSS solutions are based on SOA (Service Oriented Architecture), which dynamically discover and combine the services on a global scale to support decision-making. The main principles are:

- Services which are based on standard interfaces, utilize common data types, and are well described by standard metadata;

- Distributed computing services may be based on many interaction and transport protocols. Web services based on the HTTP protocol have so far proved to be the usable and interchangeable means of providing access to data and processing resources in a globally federated and diverse environment.

Since 2007 GEOSS has developed the Architecture Implementation Pilots (AIP) [14], which concerns with the development and pilot experiment of new process and infrastructure components for the GEOSS Common Infrastructure (GCI) and the broader GEOSS architecture through an evolutionary development process. The tools and applications developed through various research projects may be registered as components and services into the GEO Portal. The GEOSS Components and Services Registry provides a formal listing and description of all the Earth observation systems, data sets, models and other services and tools that together constitute the Global Earth Observation System of Systems. These various components are being interlinked using standards and protocols that allow data and information from different sources to be integrated. The components and services listed on the Registry can be searched and explored by decision-makers, managers and other users of Earth observations via the GEO Portal.

The GEOSS Components and Services Registry is the main GEOSS catalogue. The GEOSS Standards and Interoperability Registry enable contributors to GEOSS to configure their systems so that they can share information with other systems. One of the key components for interoperability in the GEOSS 
architecture is the clearinghouse. Clearinghouse catalogue client specifies that, in order to perform search and discovery of external (to GEOSS) resources, the GEOSS catalogue(s) should operate as discovery brokers. GEOSS Clearinghouse utilizes the OGC Catalogue Service protocol to access the Component and Service Registry and external community catalogues. On the base of analysis, GEOSS defines multiple Web Services interface Implementation Specifications based on OGC Web Services, OGC Web Map Service (WMS), Web Feature Service (WFS), and Web Coverage Service (WCS) [15]. The goal of GEOSS is to develop a dynamically interoperable infrastructure.

The enviroGRIDS project has developed and registered into GEOSS the tools and applications such as gSWAT, GreenLand, BASHYT, eGLE, and URM related services.

\section{BSC-OS PORTAL}

The BSC-OS portal is the main way of users to access the resources of the enviroGRIDS projects such as environmental data, geospatial services, hydrological models, environmental scenarios, tools and applications, distributed processing tools, satellite image processing applications, geospatial data visualization tools, environmental reports, and training materials (Figure 1). All these functional resources are implemented and provided by the Geospatial and Grid Services level.

The user accesses the portal by Web applications and published by the End User Application level [16]. Each application provides a graphical user interface with high usability. There are five categories of users with different accessing rights according with their professional qualification and assigned role. They are data providers, earth science specialists, decision makers, citizens, and system administrators. Each user may authenticate either locally for a particular application, or globally for all tools and applications within the BSC-OS portal.

The main objective on developing the end user applications was to provide the user the possibility to access from a low performance computation computer, such as his laptop, the processing of huge data on high performance computing resources (Figure 2). The application by its graphical user interface hides the complexity of managing huge distributed spatial data and computing infrastructures. The user accesses the system similarly to a simple local application. Another aim was to access distributed data repositories through a standard manner such as OGC Web Services.

The main categories of end user applications and platforms implemented and published by the portal are the followings:

- Data Management - provides the user with spatial data management and operations. The user may enter data and metadata, visualize, modify, update, and remove spatial content from data repositories. The URM platform supports the main functionality required by the end user tools and applications;

- SWAT Hydrological Model Management - provides the Earth Science specialists with hydrologic model configuration, model calibration, and hydrologic scenario running. One of the water quality models used in the enviroGRIDS project is SWAT. This model is designed to estimate impacts of land management

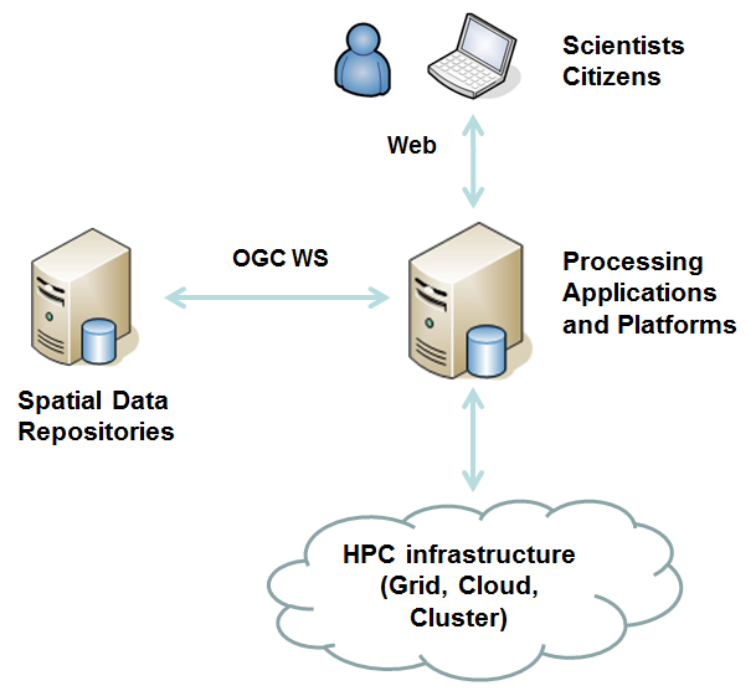

Figure 2. Web applications use remote high performance computation

practices on water quantity and quality in complex watersheds. The SWAT model requires specific information about weather, soil properties, topography, vegetation, and land management practices of the watershed. The gSWAT platform provides the main functionality required on the Web server. The gSWAT Sim platform is a set of services publishing the granular functionality of the gSWAT platform. The platform is able to manage as well the HEC-HMS hydrological models;

- $\quad$ Satellite Image Processing application and GreenLand platform - the specialists may process satellite data and images in order to search for relevant information (e.g., land cover, vegetation, water, land use, soil composition, etc);

- Geospatial Data Visualization and BASHYT platform - the specialists visualize various spatial data in different formats and views and compose environmental reports for decision makers and citizens;

- Flood Scenarios and Flood Portals - provide the decision makers with interactive and graphical tools to access data, maps, reports, and scenarios regarding the floods;

- $\quad$ eLearning Material Execution application and eGLE platform - supports the specialists to develop Earth Science oriented training materials and the regular users to execute the lessons and Earth Science related processing.

The regular users visualize the reports generated by the specialists as results of executing environmental scenarios. The input data for the reports are built up by the specialists by running hydrological models of the Black Sea catchment area and by processing related satellite data. All data sets required for building up the hydrological models, environmental scenarios, and spatial models are provided and entered into the system by data providers.

The interoperability between various platforms is supported 
by the standard OGC services [15]. The distributed processing is supported by the Grid Computing Infrastructure, based on the gLite middleware, and involving Storage Elements and Computing Elements throughout the Grid [17]. The gLite is lightweight middleware for Grid computing developed through the EGEE project as the foundation of its globally distributed computing infrastructure. Now, the middleware components in gLite became part of the EMI (European Middleware Initiative) distribution [18] and are managed as independent projects providing software to Grid infrastructures such as EGI (European Grid Infrastructure) [19].

\section{Data Management Tools}

The URM (Uniform Resource Management System) platform [20] supports the sharing, searching and fetching of spatial and non-spatial data, and establishes a network that promotes the GEOSS concept of data sharing for a more sustainable environment. URM Geoportal is a set of modules and services, which are able to communicate through interoperable services defined by OGC (Open Geospatial Consortium), and W3C (World Wide Web Consortium). The URM Geoportal consists of four basic modules interconnected through metadata:

1) Metadata management - supported by the MicKa toolset for editing and management of metadata for spatial information, Web services, and other sources;

2) Data management - supported by the DataMan application. It provides the import, export, and management of spatial data in files or databases for both raster (IFF/GeoTIFF, JPEG, GIF, PNG, BMP, and ECW) and vector (ESRI Shapefile, DGN, DWG, and GML) data types;

3) Data visualization - provided through the MapMan software tool. It supports publication of compositions from locally stored geospatial data with external WMS (Web Map Service) and WFS (Web Feature Service) data services;

4) Content management - is supported by the SimpleCMS toolset for publishing in context and connections with social networks.

\section{Hydrological Model Management}

One of the main tasks of the enviroGRIDS project is to study environmental scenarios by experimenting hydrological models for the Black Sea catchment region. The Black Sea catchment region needs a high resolution model and involved huge quantity of geospatial data sets. Therefore, the execution on a standalone computer is not efficient at all, especially for the calibration phase. The basic solution proposed through the project is to use the distributed and parallel processing over the Grid infrastructure. The project has experimented on the Grid two model types: SWAT models for Danube River and for Black Sea catchment area, and HEC-HMS for Somes Mare basin.

\section{A. SWAT Hydrological Models}

The enviroGRIDS project aims to build up, calibrate and execute huge SWAT models [3] for the Black Sea catchment region [21]. The model allows specialists to develop and study different scenarios, and to make predictions on the impact of management decisions on water, sediment, nutrient and pesticide yields with high accuracy on large river basins.

A good hydrological model is achieved by three steps: development, calibration and evaluation. The model calibration aims to select the best values for model parameters so that the real hydrological behaviour can be simulated. Most hydrological models have two types of model parameters, called physical parameters that represent physical properties of the catchment, which can be measured, and process parameters that represent characteristics which cannot be measured. The calibration process aims to minimize an objective function, which measures the difference between the simulated output of the hydrological model and the measured output. The calibration is a very expensive process requiring high performance computation resources. To reduce the costs, the enviroGRIDS project develops and experiments the calibration and execution of the SWAT model over the Grid infrastructure and evaluate the efficiency of such a solution.

One of the first models built in enviroGRIDS is the Danube model that covers an area of $801,093 \mathrm{~km}^{2}$, for a river flow distance of $2,826 \mathrm{~km}$. The region has been divided in 1,224 smaller sub-basins. The model has $1.6 \mathrm{~GB}$ (compressed) and more than 327,000 files. The calibration process requires running a high number of iterations, each iteration consisting in a high number of simulations. Since the great number of simulations are executed in parallel and distributed over the Grid, the overall execution time of one iteration is dramatically reduced compared with a sequential execution. For instance, the execution over Grid takes 21 hours for 24 simulations, with execution time per simulation of 2,586 sec. It takes 26 hours for 100 simulations, and $939 \mathrm{sec}$ per simulation, and 30 hours for 500 simulations, and that means $215 \mathrm{sec}$ for each simulation. When the system executes a high number of simulations, through distributed and parallel processing over Grid, the average execution time for one simulation is extremely short. Therefore the execution over the Grid becomes efficient for huge models that require very extensive computation scalable to a great number of users, execution processes, and data models.

Another very extensive SWAT model is that of the Black Sea catchment region covering 2.3 million $\mathrm{km}^{2}$, with rivers from 23 countries, and 160 million inhabitants. The catchment region has been divided in 12,982 sub-basins, for river length of $20,343,825 \mathrm{~km}$. The model has $1,300,000$ input output files. The calibration through 200 simulations by a sequential execution on a standalone machine could take 8,059 hours, while the Grid based distributed and parallel calibration manages to reduce the time to 40 hours.

\section{B. gSWAT Application}

The gSWAT application has been developed in enviroGRIDS project and is available through the BSC-OS portal in order to support the calibration and execution of the SWAT models [22], [23]. The Grid infrastructure is the basic solution for parallel and distributed processing of the hydrological model by the gSWAT application [21]. It is developed as a Web application that hides to the user the complexity of the Grid infrastructure (Figure 3). The application provides support 


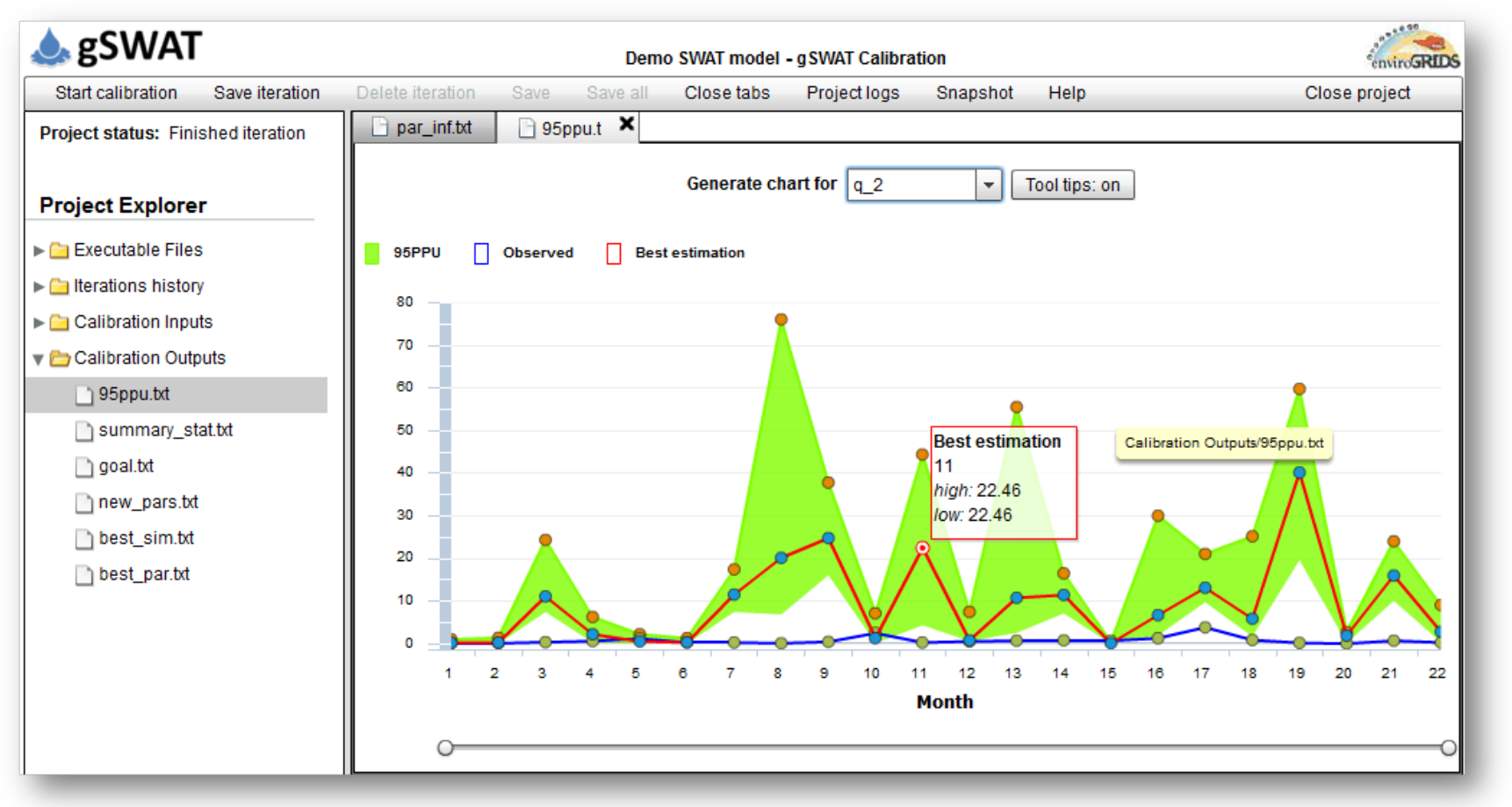

Figure 3. Graphical visualization of the SWAT model calibration results

for scalable models in terms of geographical area, modeling resolution, number of models simultaneously running, and number of users. Cloud [24], Multicore architecture [25], and GPU cluster based solutions are explored as well in order to speed up and optimize the hydrological model processing.

\section{SWAT Oriented Services}

gSWATSim is a server side extension of the gSWAT platform that is exposed as a collection of REST Web Services supporting the user to create new projects and scenarios; run environmental scenarios; modify some project related information such as name, description, etc.; upload results to visualization module like BASHYT; and get the execution status of scenarios.

\section{SWAT Model Development and Running}

The hydrological model could be developed, calibrated and run through various approaches based on the gSWAT, gSWATSim, and BASHYT platforms. The specialist could choose one of the following solutions:

1) gSWAT Application: The environmental specialist develops the SWAT model by using ArcSWAT and ArcView tools on his computer. By using the gSWAT application the user uploads the model onto the gSWAT server and executes interactively the calibration of the model [22]. The user controls the steps to reach the optimal calibration by setting up the parameters, the simulations, and the iterations, through user interactive techniques provided by the Web graphical user interface. For instance, in Figure 3 the user visualizes graphically the set of executed simulations and the best one. Finally the user can download the resulted calibrated model.
2) gSWAT and BASHYT Tools: The gSWAT and BASHYT applications collaborate through different working sessions that are connected just at the data level. The main advantage of this solution is the portability of the data model between independent tools. The user carries out the following steps:

1) Develops the SWAT model just in BASHYT;

2) Downloads the archived SWAT files and metadata onto the Storage Element accessed by the gSWAT platform;

3) Performs the calibration by gSWAT as in the first solution;

4) Uploads the results into BASHYT and visualizes the environmental information.

3) gSWATSim Services: The applications work together through a common Storage Element and dedicated Web Services. The control flow of the processing is in BASHYT through which the user develops the model and defines the scenario. The user exports model data onto the Storage Element by gSWATSim services. Then through dedicated Web Service the user customizes the execution environment, and performs the execution of the scenario. The progressing of the scenario execution can be sampled from BASHYT. Finally, after the execution is completed, the results are available automatically into BASHYT for visualization. By this solution the user does not need to switch between the applications. BASHYT accesses a new functionality available through gSWATSim services, which allows both the execution and the monitoring of running scenarios. 


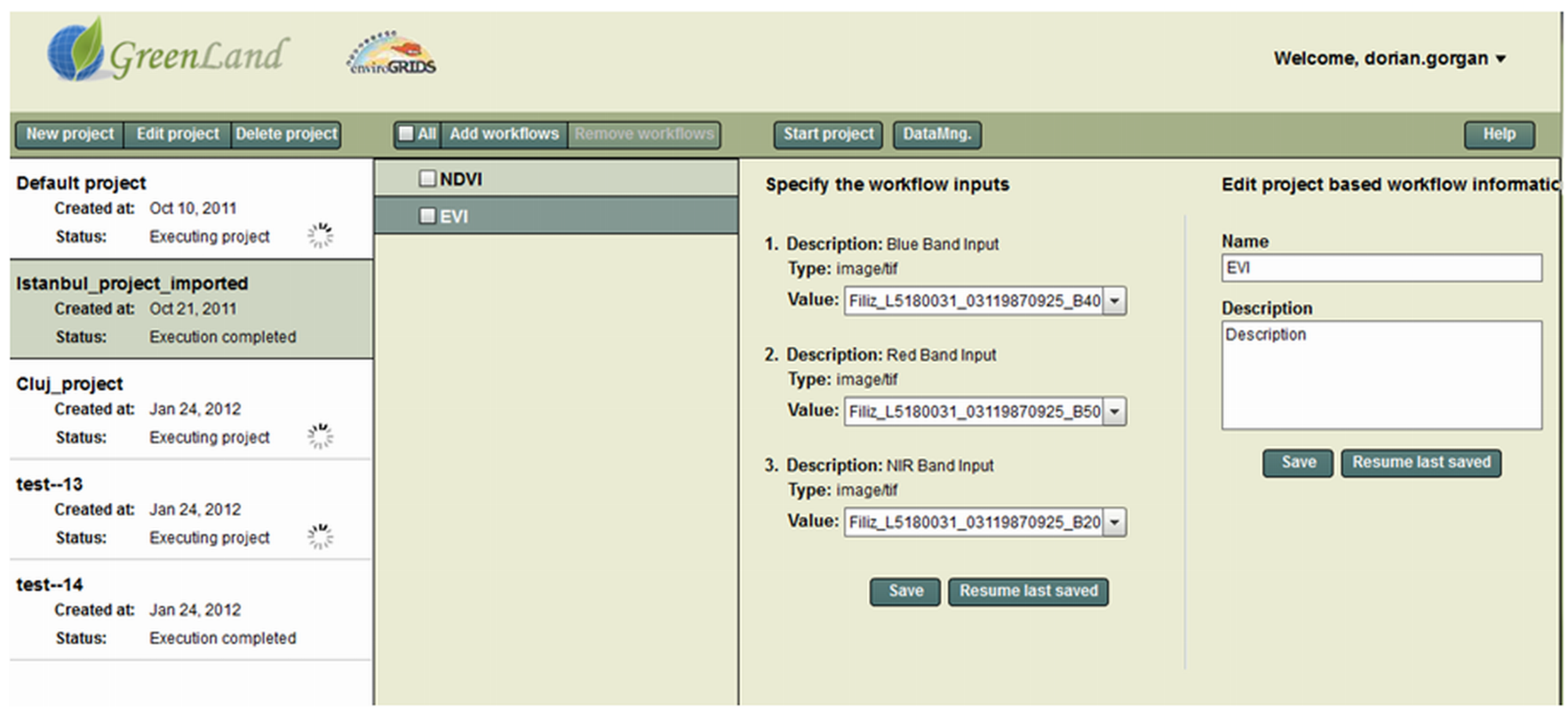

Figure 4. GreenLand application available in the BSC-OS portal for satellite image processing over the Grid infrastructure

\section{SAtellite Image Processing}

The satellite image processing is supported in the BSC-OS portal by the GreenLand platform and application. Satellite images such as MODIS and Landsat, could reveal information on land cover, precipitations, soil composition, moisture, pollution, and various natural phenomena. Spatial and environment related data could be obtained by imagery classification and processing of the multispectral bands. The image classification is a multivariable process that requires flexible and powerful tools and applications to support an optimal search for the appropriate solutions.

The GreenLand platform supports the development of Grid based applications for satellite image processing, and layers the ESIP (Environment oriented Satellite Data Processing Platform) and gProcess platforms [26]. ESIP supports a workflow based flexible description of the satellite images complex processing over the Grid. the ESIP platform includes the gridified GRASS library [27]. The gProcess platform supports the management and execution of workflows, task distribution, and management of parallel and sequential tasks across the Grid infrastructure.

The BSC-OS portal publishes the GreenLand end user application that is accessible by Web browsers (Figure 4). The GreenLand application offers the following satellite image processing related functionalities:

- Describe the image processing by acyclic graphs. There are two types of graphs: (a) pattern description called PDG (Process Description Graph), and (b) instantiated description called iPDG (Instantiated PDG). PDG describes the processes by basic operators, services, subgraphs, data types, and their connectivity throughout the graph. iPDG completes the PDG description by real data that have to be processed;

- Describe the basic functionality by a set of basic operators. The complex functionality is described by workflows, and remote Web services;

- Complex processing is executed in parallel and distributed over the Grid infrastructure. The simple processing is executed locally if it requires low performance computation resources;

- gProcess platform maps the workflow description onto the physical resources of the Grid infrastructure;

- Supporting the scalability, in terms of number of users, number of projects, number of workflows;

- GreenLand uses OGC Web services to search, visualize, fetch, and store the satellite images;

- Interoperability between GreenLand and URM is supported by standard OGC services (e.g., WMS, WCS, and WFS);

- GreenLand publishes satellite data by OGC services provided by GeoServer, and registered on the URM server;

- GreenLand functionality and operators are published through a standard WPS (Web Processing Service) interface (e.g., NDVI, EVI, and Accuracy Assessment);

- Two graphical editors support the development of basic operators and workflows. The first editor includes into the GreenLand platform the basic operators, which are used later to develop complex functionalities as workflows. The workflow editor supports the diagrammatic description of complex processing to be executed over the Grid.

Through the enviroGRIDS project the features of the GreenLand have been extended to cover the requirements of three main use cases: (a) land cover monitoring for the Istanbul area in Turkey, (b) Rioni River in Georgia, and (c) Mosaic scenario related with the Black Sea catchment region. 


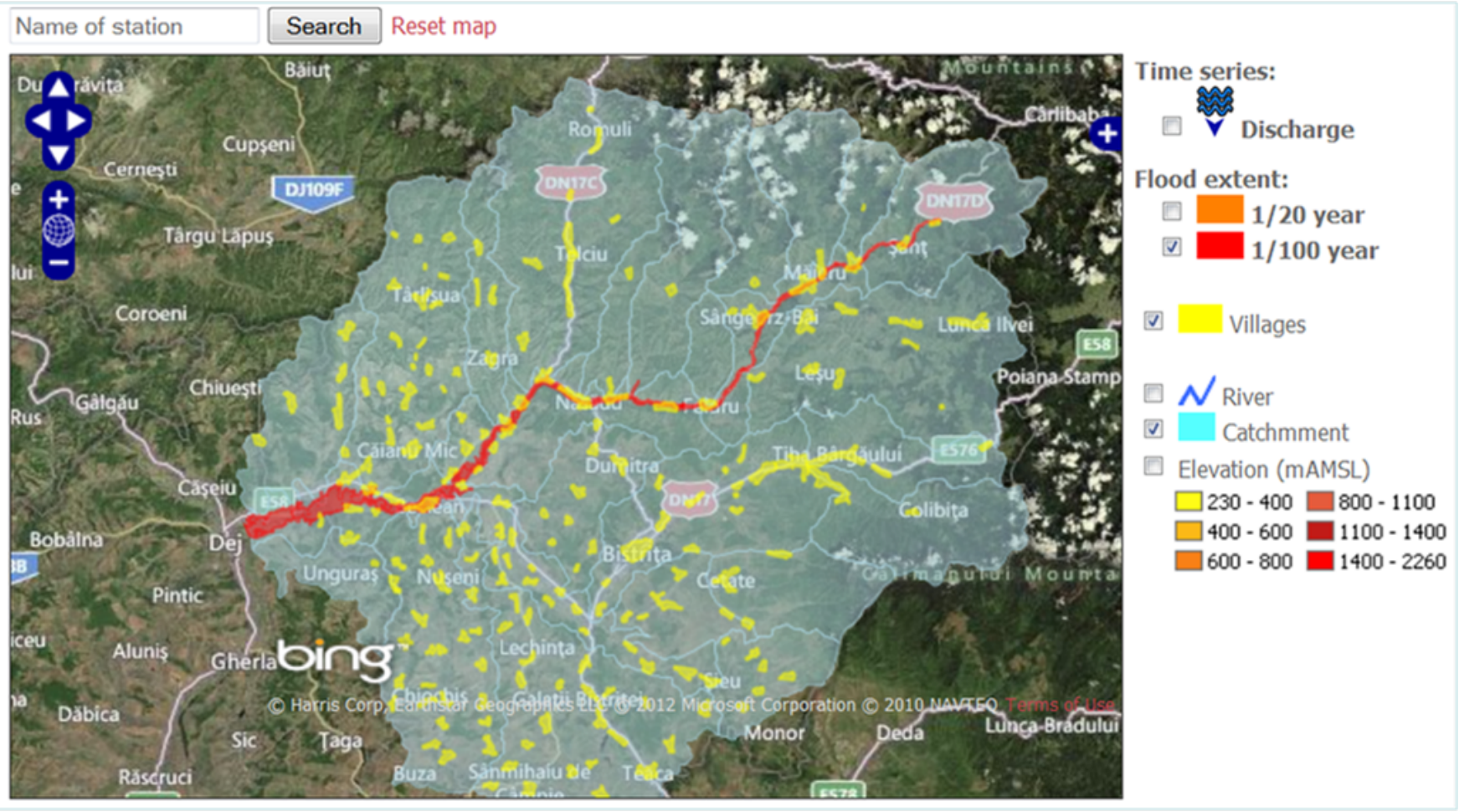

Figure 5. Flood Portal for the Somes Mare catchment area

\section{ViII. Geospatial Data Visualization}

BASHYT (The Basin Scale Hydrological Tool) is a Web based interface to SWAT that works together with ArcSWAT and AvSWAT [28]. It can be used to manage many watersheds/scenarios at once and exposes on the Web a template to produce environmental applications. BASHYT supports adaptive strategies for water and soil resource vulnerability. The tools assist decision makers in the field of sustainable water resources management. Such a decision support system is designed to meet the needs of administrations involved in integrating environmental reporting procedures (based primarily on GIS, tables, graphs) and analysis tools. BASHYT supports a Web based, live programming environment, making the programming features available to developers with almost no learning curve. This increases the productivity of the software development by reducing scaffolding code of end user applications.

In BASHYT the SWAT models are stored into a relational database, and a preprocessing step is required to import raw data (vector, raster and tabular data) into the system. After importing SWAT models BASHYT could offer tables, charts, and maps in a transparent way to the end users.

\section{Flood SCENARIOS}

Two demonstrator Web applications, available through the BSC-OS portal, have been developed for citizens within the enviroGRIDS project. The first application, which is related to near real time dissemination of environmental data to citizens, a flood forecasting demonstrator is applied on the Somes Mare catchment in north-western Romania (Figure 5). For the second application, related to long term planning in river basins a demonstrator for long term planning of remediation strategies regarding flooding, sediment and ecosystem problems along the Danube River section between the towns of Braila and Isaccea has been selected.

The first application is supported by the HEC-HMS [5] hydrological model calibrated over the Grid infrastructure. For the calibration the computation system executes a large number of iterations of HEC-HMS model with randomly generated parameters. The model is developed for the Somes Mare catchment area of $5,078 \mathrm{~km}^{2}$, covering 27 sub-basins, along a length of $136 \mathrm{~km}$. Grid calibration consists of 1,000 iterations executed on 6.72 hours, over 286 worker nodes, and generating $1 \mathrm{~TB}$ of data results.

The second use case is supported by the SOBEK $1 \mathrm{D} / 2 \mathrm{D}$ [6] hydrodynamic model of flow and sediment transport. Geospatial data are available through the enviroGRIDS URM Portal by standard OGC services, while for water-related time series data the emerging WaterML standard is used.

On the client side, for both applications the main interfaces are map-based such as OpenLayers, Google maps, and Google Earth platforms, over which the additional data are overlaid as spatially distributed data, or point data containing time series of modeled results.

\section{Training Material Development And EXECUTION}

The BSC-OS portal provides access to the virtual training center based on eGLE (GiSHEO eLearning Environment) [29]. Both the authoring and the execution of the training materials are supported by eGLE. The user plays two roles called generically teacher and student. 


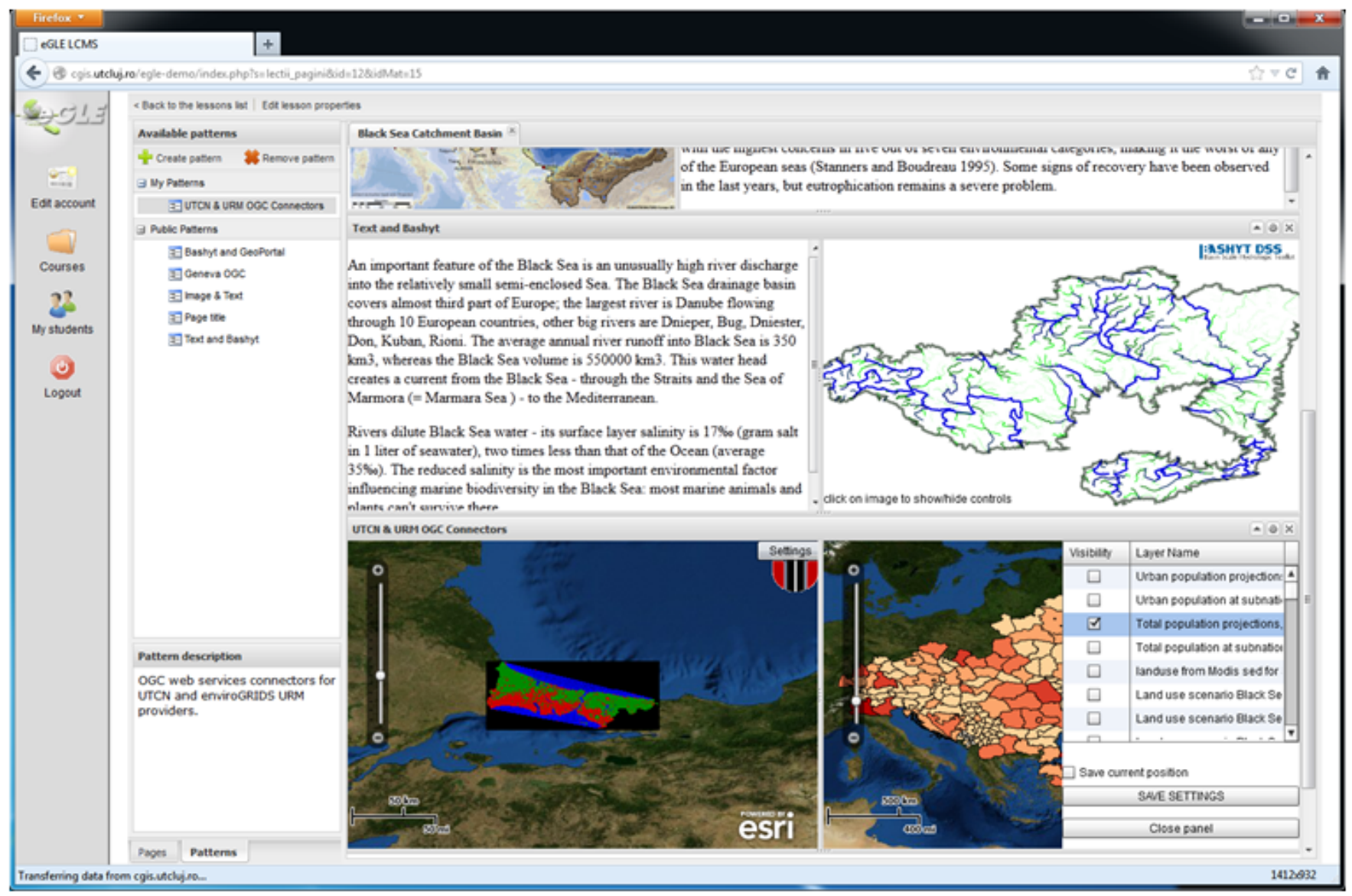

Figure 6. eGLE eLearning platform for teaching materials development and execution

The teacher is the Earth Science specialist who authors teaching materials and coordinates the training sessions. The student is the trainee who accesses the teaching objects organized within lessons in order to get presentations, experiment algorithms on spatial data, process satellite images, execute environmental scenarios, and visualize reports already prepared by the specialists.

The teaching material is organized by lessons in terms of structural templates, patterns, and tools. The Earth Science related content of the lessons may be static or dynamically fetched from data repositories by standard OGC services such as WMS and WCS (Figure 6).

The Grid based processing provided by gSWATSim and GreenLand platforms through Web services can be included and called from the lesson content. The teacher may use the Grid based execution to process satellite images, to execute specific algorithms through workflow descriptions or to visualize previously created teaching resources such as already processed satellite images, geographical maps, diagrams, algorithm workflow descriptions, etc. The students have only the right to execute the lessons according to the constraints established by the teacher. Depending on the specified level of interaction, the students could be allowed to describe and experiment new workflows (i.e. algorithms, scenarios) or choose different input data (e.g., satellite images, parameters) for the current workflow.

\section{Conclusions}

The execution of the enviroGRIDS project in general, and the development of the BSC-OS portal in particular, have faced with many challenges and issues regarding the large spectrum of concepts, methodologies, standards, technologies, and practical solutions as well.

One of the main issues is the development of the dedicated SDI, by gathering data from different sources, countries, formats, resolution, consistency, and correctness in order to fit them for the same purpose. The unitary management of huge geospatial data sets involved in the development of hydrological models and environmental scenarios (e.g., Danube, Mosaic, Black Sea Catchment, Istanbul use case) over the Grid has been a challenge indeed.

Another challenge has been the interoperability between Geospatial and Grid infrastructures, which are conceptually and technologically different in terms of security and user access rights and policy, service and tasks based granularity of the software components, flow control of processes and processing, management of data repository and resulted data. An important issue is the connectivity through standard OGC services, and interoperability between different platforms developed by the project partners (e.g., URM, gSWAT, ESIP, gProcess, GreenLand, gLite, BASHYT, and eGLE).

The portal development has to keep compatibility with new technologies and functional requirements. One main concern is 
the compatibility with the new European Middleware Initiative (EMI), which aims to improve and standardize the dominant existing middlewares in order to produce one simplified and interoperable middleware [18]. EMI attempts to unify a few Grid platforms such as ARC, gLite, Unicore and dCache. The EMI and Globus platforms will empower the EGI (European Grid Infrastructure) with more stable, useable and manageable software.

The service oriented architecture, multicore, GPGPU based systems, Cloud processing are other technologies that are to be explored in order to extend the scalability, interoperability, standard connectivity, functionality, usability of end user applications, system efficiency, and to improve the performance of data processing.

The BSC-OS portal has to be able to work or to move the applications and data onto new high performance computing infrastructure such as Cloud [24]. Such attempt is the Helix Nebula project [30]. The project aims to prepare the way for the development and exploitation of a Cloud Computing Infrastructure, initially based on the needs of European ITintense scientific research organizations, while also allowing the inclusion of the needs of other stakeholders such as governments, businesses and citizens. The Cloud Computing Infrastructure as a partnership across academia and industry is working to establish a sustainable European cloud computing infrastructure, supported by industrial partners, which will provide stable computing capacities and services that elastically meet demand.

The enviroGRIDS project provides through its BSC-OS portal and the services published through the GEO Portal the main its achievements consisting of a huge repository of spatial data regarding the Black Sea catchment region, two calibrated SWAT hydrological models of Danube River and Black Sea catchment, the gSWAT application and gSWATSim services for calibrating new SWAT models, the GreenLand application to process satellite images, the BASHYT platform for data visualization, and the eGLE platform for eLearning purposes.

\section{ACKNOWLEDGMENT}

This research has been supported by the FP7 enviroGRIDS Project (Black Sea Catchment Observation and Assessment System supporting Sustainable Development), funded by the European Commission, between 2009-2013, through the Contract 226740.

\section{REFERENCES}

[1] enviroGRIDS project- Black Sea Catchment Observation and Assessment System supporting Sustainable Development, http://www.envirogrids.net/

[2] The Full Picture, GEO Group on Earth Observation, Geneva, Switzerland, pp. 278, 2007. http://www.earthobservations.org/documents/the_ full_picture.pdf

[3] Soil and Water Assessment tool - SWAT, http://swatmodel.tamu.edu

[4] K.C. Abbaspour, J. Yang, I. Maximov, R. Siber, K. Bogner, J. Mieleitner, J. Zobrist, R. Srinivasan, Spatially-distributed modelling of hydrology and water quality in the pre-alpine/alpine Thur watershed using SWAT. Journal of Hydrology, 333, pp. 413-430, 2007.

[5] Hydrologic Engineering Center - Hydrological Model System, HECHMS, http://www.wrc-hec.usace.army.mil

[6] Deltares Systems - SOBEK Suite, http://www.deltaressystems.com/ hydro/product/108282/sobek-suite
[7] European Commission, Research and Innovation Environment, 2012 http://ec.europa.eu/research/environment/

[8] DRIHM - Distributed Research Infrastructure for Hydro-Meteorology http://www.drihm.eu/

[9] ENVRI - Common Operations of Environmental Research Infrastructures, http://envri.eu/

[10] LifeWatch European research infrastructure, http://www.lifewatch.eu/

[11] D. Gorgan, V. Bacu, D. Mihon, T. Stefanut, , D. Rodila, P. Cau, K Abbaspour, G. Giuliani, N. Ray, A. Lehmann, Software platform interoperability throughout enviroGRIDS portal, IEEE Journal of Selected Topics in Applied Earth Observations and Remote Sensing (JSTARS), 5(6), pp. 16171627, 2012.

[12] GEO Portal, http://www.geoportal.org

[13] Global Earth Observation System of Systems, http://www. earthobservations.org/geoss.shtml

[14] GEOSS AIP Architecture, pp. 49, Feb. 2013 http://www. earthobservations.org/documents/cfp/201302_geoss_cfp_aip6_ architecture.pdf

[15] Open Geospatial Consortium, OpenGIS Web Service Common Implementation Specification, pp.153, 2007.

[16] D. Gorgan, V. Bacu, D. Mihon, D. Rodila, T. Stefanut, K. Abbaspour, P. Cau, G. Giuliani, N. Ray, A. Lehmann, Spatial Data Processing Tools and Applications for Black Sea Catchment Region. International Journal of Computing - IJC, Vol.11 (4), pp. 327-335, 2012.

[17] gLite - Lightweight Middleware for Grid Computing, http://glite.cern. $\mathrm{ch} /$

[18] EMI - European Middleware Initiative, http://www.eu-emi.eu/

[19] EGI - European Grid Infrastructure, http://www.egi.eu/

[20] K. Charvat, S. Kafka, M. Splichal, M. Alberts, URM for agriculture, environmental education, and knowledge sharing. WCCA 2008 - 6th World Congress on Computers in Agriculture, Tokyo, Japan, (24 - 27 August, 2008), pp. 455-460, 2008.

[21] D. Gorgan, V. Bacu, D. Mihon, D. Rodila, K. Abbaspour, and E. Rouholahnejad, Grid based calibration of SWAT hydrological models, Journal of Nat. Hazards Earth Syst. Sci., Vol. 12/7, pp. 2411-2423, 2012.

[22] V. Bacu, D. Mihon, D. Rodila, T. Stefanut, D. Gorgan, Grid Based Architectural Components for SWAT Model Calibration. HPCS 2011 International Conference on High Performance Computing and Simulation, pp. 193-198, 2011.

[23] D. Mihon, V. Bacu, D. Rodila, T. Stefanut, K. Abbaspour, E. Rouholahnejad, D. Gorgan, Grid Based Hydrologic Model Calibration and Execution. Chapter in the book: Advanced in Intelligent Control Systems and Computer Science, Dumitrache I. (Ed.), Springer-Verlag, Vol. 187, pp 279-293, 2012.

[24] L. Biro, V. Bacu, D. Rodila, L. Barabas, D. Gorgan, Grid to cloud migration of scientific applications, using dynamically created cloud clusters, IEEE International Conference on Intelligent Computer Communication and Processing (ICCP), pp.335-340, 2012.

[25] D. Rodila, V. Bacu, D. Gorgan, Comparative Parallel Execution of SWAT Hydrological Model on Multicore and Grid Architecture, International Journal of Web and Grid Services, Vol. 8, No. 3, 2012, pp. 304-320, 2012.

[26] D. Gorgan, V. Bacu, D. Rodila, F. Pop, D. Petcu, Experiments on ESIP - environment oriented satellite data processing platform, Earth Science Informatics Journal, Springer, Vol.3/4, pp. 297-308, 2010.

[27] GRASS GIS - Geographic Resources Analysis Support System project, 2011. http://grass.osgeo.org/

[28] S. Manca, C. Soru, P. Cau, G. C. Meloni, M. Fiori, Facing issues of water and soil resources vulnerability: A multimodel and multiscale, GIS - oriented Web framework approach based on the SWAT model, Proceedings of the 2009 SWAT International Conference, Boulder, USA, pp. 91-100, 2009.

[29] D. Gorgan, T. Stefanut, V. Bacu, Grid based Training Environment for Earth Observation. Advances in Grid and Pervasive Computing, LNCS Vol. 5529, pp 98-109, 2009.

[30] Helix Nebula - the Science Cloud, http://www.helix-nebula.eu/ 